\title{
Producción de conocimiento \\ y formación de investigadores
}

Un análisis de las tesis de maestría
en comunicación en México (1996-2000)

Gabriela de la Torre Escoto Raúl Fuentes Navarro ${ }^{2}$

El problema contemporáneo para los estudios de comunicación no es primordialmente la cuestión de cambiar o generar una justificación epistemológica [...] Lo que obstaculiza más bien el desarrollo académico de la comunicación proviene de las prácticas políticas, institucionales y pragmáticas de la "producción de conocimiento" en la Academia occidental del siglo XX [...] El conocimiento se ve o como una cadena de bits de información o como el poder técnico para modificar el entorno [...] Estos estándares académicos pueden basarse conceptualmente en una epistemología del siglo XVII, pero fueron reificados en la formación política del siglo XVIII que se mantiene hasta hoy

Condit, 1989: 154).

En este trabajo se expone una revisión crítica de las tendencias recientes de
desarrollo del campo académico de la comunicación en México, ${ }^{3}$ especial-
mente de sus determinaciones y manifestaciones institucionales, entre las

1. Este trabajo es un subproducto parcial del Proyecto de Bases de Información La investigación académica de la comunicación en México, 1950-2000, apoyado y financiado por el Conacyt (R34247-S) bajo la responsabilidad de Raúl Fuentes Navarro; y de la tesis de maestría en comunicación con èspecialidad en difusión de la ciencia y la cultura (ITESO), realizada por Gabriela De la Torre y dirigida por Raúl Fuentes Navarro.

2. Departamento de Estudios Socioculturales, ITESO. Correo electrónico: raul@iteso.mx

3. El concepto de "campo" se retoma, evidentemente, de la obra de Pierre Bourdieu (1972, $1975,1988,2000$ ), como espacio sociocultural de posiciones objetivas donde los agentes luchan por la apropiación del "capital" común. 
cuales los programas de posgrado ocupan un lugar relevante. En ese sentido, reasume y actualiza las interpretaciones alcanzadas en un estudio anteriormente publicado, en que se analizaron los procesos de estructuración/reestructuración del campo desde su constitución (Fuentes, 1998), e incorpora en ese marco los resultados de un análisis de las tesis de maestría producidas en tres programas nacionales entre 1996 y 2000 en cuanto manifestaciones de las tendencias actuales de producción de conocimiento así como de la formación de nuevos investigadores.

\section{Constitutivos institucionales del campo académico de la comunicación en México}

Conviene recordar que el campo académico de la comunicación en México, a diferencia de otras especialidades de las ciencias sociales o las humanidades, se originó y se centra en la formación profesional, que actualmente se imparte en alrededor de 200 instituciones de educación superior en el país. Su institucionalización parte, entonces, de la licenciatura y casi se limita a ella, pues ni los posgrados ni los centros de investigación ocupan cuantitativamente un lugar significativo ni tienen un papel central en el conjunto. Aunque más adelante enfatizaremos el análisis de su desarrollo más reciente, sigue siendo cierto que la existencia misma y el carácter de estos programas de investigación y posgrado dependen todavía, en buena medida, de las orientaciones del pregrado, y no siempre de sus mejores rasgos. En los más de treinta programas de maestría que operan actualmente en México se manifiesta claramente la disyuntiva disciplinaria de los estudios de comunicación y su compleja relación con la investigación, mientras que en el nivel del doctorado parece haberse inclinado hacia opciones no disciplinarias.

Los problemas de la formación de profesionales de la comunicación, múltiples y complejos, no pueden ser detallados aquí, pero cabe subrayar que la investigación, de cualquier manera, no ha sido eje, en ningún sentido, del desarrollo del subcampo educativo de la comunicación en México. A pesar de que los programas de licenciatura en comunicación comenzaron a establecerse desde finales de los años cuarenta, no fue sino hasta fines de los sesenta cuando empezaron a realizarse prácticas (muy aisladas) de investigación en ellos, y en los setenta cuando se dieron los primeros intentos de institucionalización de esta actividad, tanto dentro como fuera de los establecimientos universitarios. En marzo de 1974, Josep Rota presentaba el siguiente balance, que es el más antiguo que se puede documentar: 
Durante los últimos diez años, la mayor parte de la investigación ha sido comercial, realizada por agencias de publicidad o compañías de investigación de mercados. Desgraciadamente, los resultados de estos esfuerzos suelen ser confidenciales. Casi la totalidad de la investigación está constituida por las tesis de licenciatura de estudiantes universitarios, sobre todo del Departamento de Comunicación de la Universidad Iberoamericana. Se han escrito ahí $\mathbf{4 3}$ tesis entre 1967 y 1973. Otras se han realizado en la Universidad Nacional Autónoma de México. Pero aparte de las tesis, prácticamente no se ha hecho nada más (Rota, 1974: 56).

Un análisis bibliométrico del campo (Fuentes, 1988) confirmó el lacónico diagnóstico de Rota: sólo se incluyen en él 11 libros, 25 artículos y 4 informes de investigación inéditos hasta 1973. Para 1980, Rubén Jara pudo, con dificultades, reunir 100 estudios empíricos (la mayoría tesis) para realizar su Análisis de la situación actual de la investigación empírica de la comunicación en México, cuyas conclusiones asientan que "no existen actualmente en México las condiciones adecuadas para que se realice de manera apropiada una labor de investigación en comunicación" (Jara, 1981: 214). Las conclusiones de Rota y de Jara, en sus respectivas revisiones del "estado de la cuestión" (ambas realizadas desde el Departamento de Comunicación de la Universidad Iberoamericana), son antecedentes indispensables para cualquier análisis actualizado sobre la investigación de la comunicación en México. Ambos indican, antes que nada, la severa limitación de las infraestructuras necesarias para la práctica de la investigación en las universidades mexicanas, y constituyen el marco contextual indispensable para analizar el surgimiento de los posgrados.

Los dos programas de maestría creados a fines de los años setenta, en la Universidad Iberoamericana (estructurado por Jara) y en la Universidad Nacional Autónoma de México, evidencian en sus proyectos originales, y en los cambios de estructura y de orientación que han experimentado desde entonces, un patrón de discontinuidad y de desarticulación que, tanto en el plano institucional como en el cognoscitivo, caracterizan al campo en México, especialmente en cuanto a la práctica de la investigación, aunque tienen el mérito de haber mantenido la producción. Los demás posgrados, creados en los ochenta y noventa, no presentan un patrón muy distinto.

Aunque la Asociación Mexicana de Investigadores de la Comunicación (AMIC) se propuso, desde 1980, como una de sus prioridades "diagnosticar el estado actual" de la investigación, no fue sino hasta su IV Reunión Nacional (Guadalajara, febrero de 1987) cuando algunos de sus miembros abordaron la tarea. De ahí surgió un libro compilado por Enrique Sánchez Ruiz (1988), en que se discutieron las condiciones, tendencias y productos de la investigación mexicana en sus "primeros" treinta años. Un año después, Fuentes y Sánchez introdujeron la figura de la triple marginalidad para caracterizar a la investigación de la comunicación ("marginal" respecto a las ciencias sociales, 
éstas en el conjunto de la actividad científica y ésta en relación con las prioridades del desarrollo nacional) (Fuentes y Sánchez, 1989) y continuaron actualizando el análisis del campo en algunas colaboraciones conjuntas. Una de ellas caracteriza al periodo 1985-1990 como "de transición" para la investigación mexicana de la comunicación, partiendo de su estructura institucional de base:

Hasta 1985, prácticamente la totalidad de la investigación mexicana de comunicación se realizó en la Ciudad de México, ya fuera en centros universitarios o de otro carácter. La investigación académica estuvo mayoritariamente concentrada en la Universidad Nacional Autónoma de México (UNAM), aunque con importantes complementos en la Universidad Autónoma Metropolitana-Xochimilco (UAM$\mathrm{X}$ ), la Universidad Iberoamericana (UIA) y, durante unos años, la Universidad Anáhuac. La investigación no universitaria ha incluido centros privados, como Comunicología Aplicada de México (del grupo publicitario Ferrer) y el Instituto de Investigación de la Comunicación (filial de Televisa); otros internacionales, como el Instituto Latinoamericano de Estudios Transnacionales (ILET), el Instituto Latinoamericano para la Comunicación Educativa (ILCE) y el Centro de Estudios Económicos y Sociales del Tercer Mundo (Ceestem); se pueden incluir también algunos centros paraestatales como el Centro Nacional de Productividad (Cenapro) y el Centro de Medios y Procedimientos Avanzados de Educación (Cempae) y diversas dependencias del gobierno federal que, especialmente en los años setenta, contribuyeron de manera importante en diversas áreas del estudio de la comunicación. La crisis provocó que la mayor parte de estos centros, ubicados todos en la capital del país, disminuyeran considerablemente su producción, o cerraran (Fuentes y Sánchez, 1992: 25).

Debido a lo que comúnmente se conoció como "la crisis nacional" de los ochenta, hasta 1990, según esa figura "de transición", la proporción de la investigación realizada en la UNAM se redujo drásticamente, mientras que la de la UAM-Xochimilco se incrementó un poco; la de la UIA se sostuvo, pero la aportación de la Universidad Anáhuac se retrajo mucho, así como las de Comunicología Aplicada y el ILET. Finalmente, el Ceestem, los centros paraestatales (Cenapro y Cempae) y los formados en varias secretarías de estado y dependencias oficiales fueron víctimas, en diversos momentos, de los recortes presupuestales del gobierno federal y desaparecieron.

No obstante, en el mismo periodo se crearon nuevos centros de investigación de la comunicación en el país y se incrementaron los espacios de diálogo e interrelación tanto entre instituciones como entre investigadores, a través de reuniones de trabajo, proyectos específicos y publicaciones periódicas. Estos nuevos centros, que incorporaron a investigadores posgraduados tanto en el extranjero como en México, e impulsaron la investigación de manera muy notable desde la segunda mitad de los ochenta, son el Programa Cultura, 
fundado en 1984 y adscrito al Centro Universitario de Investigaciones Sociales de la Universidad de Colima; el Centro de Estudios de la Información y la Comunicación (CEIC) de la Universidad de Guadalajara, establecido en 1986 y transformado en Departamento de Estudios de la Comunicación Social (DECS) en 1994; y el Programa Institucional de Investigación en Comunicación y Prácticas Sociales (Proiicom), constituido en 1989 en la Dirección de Investigación y Posgrado de la Universidad Iberoamericana e incorporado al Departamento de Comunicación en 1995.

Junto a algunos de los programas de posgrado, que se revisan más adelante, estos tres centros de investigación constituyeron, en los últimos quince años, el núcleo de una práctica de investigación de la comunicación quizá por primera vez verdaderamente sistemática, colectiva y nacional, relativamente independiente de los programas de licenciatura y con proyección internacional. En algún sentido, la crisis económica de los ochenta, al mismo tiempo que desestructuró la configuración que el campo había adquirido en los setenta, propició una reestructuración aparentemente más sólida institucionalmente y más productiva académicamente, aunque muy concentrada en sólo seis instituciones. ${ }^{4}$

A partir de la primera mitad de los años noventa se detectó una tendencia clara hacia el distanciamiento entre la investigación "aplicada" o "comercial" y la académica. Los proyectos más directamente vinculados con la toma de decisiones en algunos ámbitos de las prácticas sociales de comunicación, que los norteamericanos llaman "investigación administrativa", se desplazaron decididamente hacia agencias especializadas, siguiendo el auge de los estudios de mercado y de opinión pública que trajo consigo la "modernización" económica y el "adelgazamiento" del Estado. Por su parte, la mayoría de los proyectos académicos se concentraron en la profundización "crítica" del conocimiento sobre diversas temáticas y desde distintos enfoques metodológicos (predominantemente cualitativos), aunque incrementaron su grado de desvinculación con la formación profesional de los estudiantes de comunicación.

Con la excepción del reforzamiento de algunos programas de posgrado, la investigación académica encontró nuevos espacios de desarrollo mediante relaciones más estrechas con centros, investigadores y enfoques de otras disciplinas de las ciencias sociales que con las licenciaturas en comunicación. Es muy elocuente en este sentido la orientación de los tres centros creados en los años ochenta, totalmente desvinculados organizacionalmente de las carreras profesionales, así como su destino más reciente: en la Universidad de

4. La UNAM (FCPyS e IIS), la UAM-Xochimilco, la Universidad Iberoamericana, la Universidad de Guadalajara, el ITESO y la Universidad de Colima. En ellas se produjo $70.8 \%$ de la investigación académica nacional sobre comunicación entre 1986 y 1994 (Fuentes, 1996). 
Guadalajara se articuló la investigación con los posgrados y se incrementó la planta de investigadores, pero los académicos más reconocidos del Programa Cultura de la Universidad de Colima cambiaron su adscripción a otras instituciones, y el Proiicom de la Universidad Iberoamericana se disolvió para dar espacio al desarrollo de proyectos académicos diferentes, sustentados por otros investigadores, que sustituyeron a los fundadores.

También es notable el proceso de descentralización que la investigación de la comunicación ha experimentado desde mediados de los años ochenta, no sólo por la desaparición de muchos de los núcleos institucionales que operaron antes en la zona metropolitana de la capital, sino por la instalación de nuevos centros fuera de la Ciudad de México. Por ello puede afirmarse que, aunque está lejos todavía un equilibrio entre las diversas regiones del país en términos de recursos y producción, las contribuciones provenientes de algunos estados (Jalisco, Colima, Nuevo León, Baja California, Sonora, Veracruz, Puebla, Guanajuato, entre otros) han aumentado considerablemente en cantidad y en calidad, desahogando un poco la presión que se había acumulado sobre los investigadores y los centros de investigación ubicados en la capital, para dar cuenta del panorama comunicacional nacional.

De hecho, el análisis bibliométrico de la producción del campo indica que a mediados de la década pasada podía hablarse ya del establecimiento de una estructura bipolar en la investigación académica de la comunicación en el país, pues la contribución de la región centro-occidente (o más específicamente, de Guadalajara y Colima), pasó de $1.5 \%$ de los productos publicados entre 1965 y 1974 a $12.2 \%$ entre 1975 y 1984 , y a $29.5 \%$ entre 1985 y 1994 . En esta región, igualmente, se ha llegado a editar $27 \%$ de las publicaciones nacionales en el campo de la última década y media (Fuentes, 1996, 1998). No obstante, en esta "descentralización" hacia Guadalajara y Colima se descubren dos características importantes: primero, que han sido más determinantes para su surgimiento los factores de orden nacional, e incluso internacional, que los propiamente regionales o locales, exceptuando el impulso de las autoridades universitarias correspondientes. Y segundo, que la producción de investigación "descentralizada" apenas ha abordado en $25 \%$, aproximadamente, cuestiones específicas de la comunicación y la cultura en la región en que se realiza: tres cuartas partes de esta producción siguen enfocando nacional e internacionalmente sus objetos de estudio. Estas tendencias se confirman (provisionalmente, pues el análisis no ha sido concluido a la fecha) para el periodo más reciente (1995-2001).

A partir de estos rasgos estructurales, cabe concluir con la consideración de que, en general, entre los desafíos y perspectivas de la investigación mexicana de la comunicación, se reconoce que la prioridad ha estado puesta en las condiciones que definen la profesionalidad de los investigadores: por un lado, la consolidación y ampliación de los apoyos laborales e institucionales 
necesarios para concentrar la dedicación en las tareas de desarrollo científico y académico en algunas instituciones; por otro lado, el incremento y reconocimiento de la calificación científica, especialmente en lo que corresponde a la solvencia metodológica de las investigaciones, aspecto que, hasta años muy recientes, ha sido particularmente descuidado (Fuentes y Sánchez, 1992: 35). En ambos sentidos, ha sido fundamentalmente importante el desarrollo de los programas de posgrado.

\section{Los programas de posgrado como instancias de impulso a la investigación}

En junio de 1989 se realizó en Guadalajara (ITESO) la Primera Reunión Nacional de Posgrados y Centros de Investigación en Comunicación, bajo los auspicios del Consejo Nacional para la Enseñanza y la Investigación de las Ciencias de la Comunicación (CONEICC) y la Federación Latinoamericana de Asociaciones de Facultades de Comunicación Social (FELAFACS).$^{5}$ Es muy significativo que, más de diez años después, las evaluaciones críticas de los participantes conserven la pertinencia para el análisis estructural de este aspecto del campo académico. Algunas de las reflexiones de la reunión, recogidas en la relatoría final, mantienen su actualidad:

Se constató que los posgrados no son instancias de investigación que alimenten a programas de formación, sino que surgen de la demanda y la estructura escolar. Los programas de maestría son propuestas que, viniendo desde la docencia, tienen a la investigación más como un problema que como un insumo. De ahí que sea interesante observar cómo se articula la relación docencia-investigación en cada una de las instituciones. También se enfatizó la escasez de recursos humanos calificados para la investigación.

La lógica universitaria $\longrightarrow$ institucional- condiciona el planteamiento de cada uno de los programas. Sus objetivos entran en la lógica propia de cada institución. De ahí que haya que plantear cómo entiende cada programa las necesidades sociales. Las maestrías son, en muchos casos, "puntas de lanza" de las instituciones a las que pertenecen, y se constata la manera como la comunicación sigue afectando a cotos disciplinares muy cerrados, haciendo que se abran a la interdisciplinariedad.

5. Se presentaron y discutieron los siguientes programas de posgrado e investigación: Universidad Iberoamericana, UNAM-FCPyS, U. Regiomontana, U. Autónoma de Nuevo León, ITESO, CADEC, ENEP-Acatlán (proyecto), U. de las Américas-Puebla (proyecto), Programa Cultura, CEIC-UdeG y Proiicom-UIA. De las instituciones convocadas, sólo faltaron representantes de la UAM-Xochimilco y del ITESM-Monterrey. 
Preocupa que en poco tiempo ocurra el boom de las maestrías, tal y como ocurrió con las licenciaturas, en vista de que se sabe de por lo menos cinco instituciones más que piensan abrir posgrados próximamente. Se observa que se abren centros de estudios sin investigar las necesidades a las que sus propuestas darían satisfacción. Por ello se considera conveniente evaluar la experiencia de los que ya tienen tiempo funcionando, para hacer algún tipo de pronunciamiento conjunto que retome esa experiencia y proporcione un panorama del posgrado en el país.

Se observa también que el nivel académico de la licenciatura ha bajado, por lo que, en ocasiones, se pretende que la maestría subsane sus deficiencias. Además, en otros casos las exigencias respecto a la maestría son tan altas que correspondería más a un doctorado satisfacerlas. Es conveniente señalar cuáles son los mínimos constitutivos de un programa de maestría: al hacerlo, se obligará a redefinir tanto la licenciatura como el doctorado.

Se planteó el problema de la formación universitaria versus la capacitación profesional: respecto a los supuestos éticos y sociales, ilos programas de maestría deben pretender reproducir o incidir en la transformación social?; respecto a la temática de estudio, ideben formar académicos, profesionales de la comunicación o ambos? Así mismo, se tocó la cuestión de la especialización y su relación con la independencia-dependencia para trabajar en problemas que institucionalmente no se consideran relevantes (Juárez, 1989: 7-8).

A partir de estos elementos de diagnóstico y composición, la reunión se planteó en un segundo momento la meta de "establecer cuáles son los elementos que constituyen el campo del posgrado en comunicación en México", a través de la discusión alrededor de cuestiones como:

¿A qué tipo de necesidades y prácticas sociales se orienta la formación de posgraduados en comunicación en México? ¿Cómo caracterizar los modelos curriculares y pedagógicos del posgrado en comunicación en el país? ¿Cómo se articulan los elementos educativos y las finalidades sociales? ¿Cómo caracterizar los proyectos de conocimiento de los posgrados y centros de investigación, en su relación con lo social? ¿Hacia dónde apunta la generación de conocimiento, cómo se articula con el currículum?

Aunque no pudieron elaborarse respuestas conclusivas a estas cuestiones, algunas formulaciones alcanzaron consenso entre los participantes y, como se señaló antes, son todavía representativas del estado actual de la reflexión nacional al respecto:

Es conveniente tomar en cuenta que la inserción en el espacio universitario del campo es aún emergente: su objeto de estudio no ha sido definido totalmente, junto a la devaluación de la profesión. Pero las maestrías no deben ser vistas como centros de capacitación, sino que deben ser algo más; han tendido a satisfacer las necesidades del medio pero también debieran "abrir brecha". En tanto que el 
campo busca su consolidación, se debe pasar a una posición más agresiva: valorar el capital ya existente, erigirse en órganos de consulta, es decir, monopolizar el saber para coordinarlo, pues no se reconoce socialmente a quien tiene el saber en comunicación. Por otra parte, para hacer una maestría se necesitan recursos, equipos de trabajo: docentes con posgrado, investigación, bancos de información, biblioteca especializada, equipo técnico y salidas hacia la sociedad.

También cabe revisar la adecuación de los perfiles con la situación laboral en el campo. Debiera también darse un seguimiento a los aspirantes a maestros para detectar su origen intelectual y observar qué tipo de práctica profesional realizan, cuáles son sus expectativas y aspiraciones para contrastarlas con los perfiles que tiene cada programa.

Desde el punto de vista del establecimiento de la oferta y la demanda de conocimiento en el campo, éste se caracteriza por la diversidad, la pobreza y los obstáculos y limitaciones -instrumentales y míticas - que padece. Se parte de que el conocimiento está inserto en un mercado que exige saber-hacer y conocimiento de la realidad circundante. También de que las ofertas de conocimiento se empezaron a transferir de las licenciaturas a las maestrías. La "pobreza" del campo se refiere tanto a recursos materiales como culturales; los obstáculos y limitaciones instrumentales y "míticas" refieren a las distintas concepciones de conocimiento que operan en el campo, que tienden a sobrevalorar (y subvalorar) la producción de conocimientos.

Por otro lado, hay que recordar que la infraestructura también se refiere a la cultura, lo que remite a un problema metodológico. En el trabajo se observan dos clases de vicios: lo que no se sabe hacer y lo que se sabe hacer mal. Establecer una cultura académica es clave: cómo hacer las cosas más eficientemente y bien hechas. A veces faltan recursos, pero a veces lo que falta es saber aprovecharlos (Juárez, 1989: 8-10).

El coordinador de la reunión referida sintetizó los retos de los posgrados y centros de investigación en el campo académico de la comunicación en México, considerándolos como los impulsores de "una fuga hacia arriba":

A pesar de que en este terreno se está todavía muy lejos de generar respuestas teóricas consistentes y de consolidar un trabajo a la altura de las necesidades de comprensión del objeto en cuestión, es indudable que el campo académico está experimentando un proceso importante de cambio caracterizado por la aparición de nuevos actores y proyectos, la incorporación al trabajo de otro tipo de preguntas y problemáticas y la extensión de las tareas educativas hacia niveles más altos de formación. La ampliación de fronteras del campo académico [...] genera la necesidad de un reacomodo general y una redefinición en la división social del trabajo académico, en circunstancias institucionales, científicas, sociales y laborales un tanto errátiles (Luna, 1989: 61).

La evaluación de ese "reacomodo general" del campo académico y esa "redefinición" en la división social del trabajo académico, exige el análisis de 
lo acontecido a partir de la fecha de la referida reunión, en una escala más amplia que la de los programas. La realización de cuatro reuniones nacionales más, entre octubre de 1999 y junio de 2001 (México, DF: UAM-Xochimilco; Guadalajara: UdeG; Monterrey: UdeM; y Guadalajara: ITESO), convocadas por el CONEICC, permiten actualizar el diagnóstico y las perspectivas del posgrado en comunicación en México. ${ }^{6}$

Respecto a la primera reunión (1989), en estas cuatro se hizo evidente que aumentó la necesidad de diálogo y colaboración entre los posgrados y centros universitarios de investigación, dado el relativo crecimiento del número y calidad de los programas, la maduración del CONEICC como espacio de interlocución privilegiado del campo de la comunicación en el país, y los cambios suscitados en el sistema nacional de educación superior, los entornos mexicano y mundial del estudio académico de la comunicación, y sus prácticas sociales de referencia.

Por una parte, las políticas nacionales en el ámbito de la educación superior establecieron, desde 1991 y hasta 2002, una distinción entre los programas de posgrado, mediante el Padrón de Excelencia del Consejo Nacional de Ciencia y Tecnología (Conacyt), que calificaba la "calidad académica" en función de diversos parámetros internacionales y canalizaba los apoyos sobre esta base. Los nueve programas de posgrado en Comunicación, con esa denominación u otra, más general, donde se trabajan proyectos de comunicación, incorporados en dicho padrón hasta 2001, son los enlistados en el cuadro 1.

De los otros programas de posgrado en comunicación que operan sin el reconocimiento de Conacyt, ya sea porque sus propósitos son distintos a la formación de investigadores o porque sus recursos no cumplen las condiciones impuestas como parámetros - la mayor parte de ellos adscritos a instituciones privadas y de reciente constitución-, hay que destacar a las maestrías en comunicación de la Universidad Iberoamericana y del ITESM campus Monterrey, que en años anteriores fueron reconocidas por Conacyt y que, por cambios en las respectivas políticas institucionales, dejaron de contar con tal acreditación, no obstante su continuada participación en la formación de investigadores y profesionales de muy buen nivel.

6. Aunque la asistencia de los coordinadores de los programas fue muy fluctuante en las cuatro reuniones, participaron en este proceso representantes de las maestrías de la Universidad Iberoamericana, la UNAM, la U. Autónoma de Nuevo León, el ITESO, la UIA León, la UIA Laguna, el ILCE, el CADEC (3 programas), la U. Intercontinental, el ITESM campus Monterrey, la Universidad Anáhuac ( 3 programas), la UAM-Xochimilco, la U. Autónoma de Aguascalientes, la UdeG (2 programas), la U. Veracruzana, la U. Regiomontana, la U. Autónoma de Coahuila, el Instituto Campechano, la U. de La Salle-Bajío, la UNIVA, la U. Autónoma de San Luis Potosí, la U. de Occidente-Los Mochis, y doctorados en operación o en proyecto de la UNAM, la UAM-X, la UdeG (2 programas), la U. de Colima, la U. Autónoma de Yucatán y la U. Autónoma del Estado de México. Las cuatro reuniones fueron coordinadas por Raúl Fuentes Navarro y María Martha Collignon. 


\section{Cuadro 1}

Posgrados de excelencia reconocidos por Conacyt en que se realiza investigación de la comunicación en México, 2001

\begin{tabular}{|c|c|}
\hline Institución & Programa \\
\hline $\begin{array}{l}\text { Instituto Tecnológico y de Estudios Superiores de } \\
\text { Occidente. Departamento de Estudios Socioculturales }\end{array}$ & $\begin{array}{l}\text { Maestría en Comunicación con } \\
\text { especialidad en difusión de la ciencia } \\
\text { y la cultura. }\end{array}$ \\
\hline $\begin{array}{l}\text { Universidad de Guadalajara. Centro Universitario de } \\
\text { Ciencias Sociales y Humanidades. Departamento de } \\
\text { Estudios de la Comunicación Social. }\end{array}$ & Maestria en Comunicación. \\
\hline $\begin{array}{l}\text { Universidad de Guadalajara. Centro Universitario de } \\
\text { Ciencias Sociales y Humanidades. Departamento de } \\
\text { Estudios de la Comunicación Social. }\end{array}$ & $\begin{array}{l}\text { Maestría en Ciencias Sociales. } \\
\text { Especialidad en Comunicación Social. }\end{array}$ \\
\hline $\begin{array}{l}\text { Universidad Autónoma Metropolitana-Xochimilco. } \\
\text { División de Ciencias Sociales y Humanidades. }\end{array}$ & Maestría en Comunicación y Política. \\
\hline $\begin{array}{l}\text { Universidad Nacional Autónoma de México. Facultad de } \\
\text { Ciencias Políticas y Sociales-Instituto de Investigaciones } \\
\text { Sociales. }\end{array}$ & Maestria en Comunicación. \\
\hline $\begin{array}{l}\text { Universidad de Guadalajara. Centro Universitario de } \\
\text { Ciencias Sociales y Humanidades. Departamento de } \\
\text { Estudios de la Comunicación Social. }\end{array}$ & $\begin{array}{l}\text { Doctorado en Ciencias Sociales. } \\
\text { Linea de Medios de Difusión e } \\
\text { Industrias Culturales. }\end{array}$ \\
\hline $\begin{array}{l}\text { Universidad de Guadalajara. Centro Universitario de } \\
\text { Ciencias Sociales y Humanidades. Departamento de } \\
\text { Estudios de la Comunicación Social. }\end{array}$ & $\begin{array}{l}\text { Doctorado en Educación. Área de } \\
\text { Comunicación y Educación. }\end{array}$ \\
\hline $\begin{array}{l}\text { Universidad Autónoma Metropolitana-Xochimilco. } \\
\text { División de Ciencias Sociales y Humanidades }\end{array}$ & $\begin{array}{l}\text { Doctorado en Ciencias Sociales. Área } \\
\text { de Concentración en Comunicación y } \\
\text { Política. }\end{array}$ \\
\hline $\begin{array}{l}\text { Universidad Nacional Autónoma de México. Facultad de } \\
\text { Ciencias Politicas y Sociales-Instituto de Investigaciones } \\
\text { Sociales. }\end{array}$ & $\begin{array}{l}\text { Doctorado en Ciencias Politicas y } \\
\text { Sociales. Orientación en Ciencias de } \\
\text { la Comunicación. }\end{array}$ \\
\hline
\end{tabular}

Fuente: Conacyt (http://www.main.conacyt.mx/daic/padron-excel.html)

Las mismas políticas nacionales, a partir de 1984 mediante el establecimiento del Sistema Nacional de Investigadores (SNI), han fijado los parámetros de reconocimiento y apoyo oficial a los académicos dedicados a la investigación y, por lo tanto, a las instituciones en que trabajan. El número de investigadores de la comunicación reconocidos por el SNI ha crecido sustancialmente en los últimos diez años, aunque su número es todavía muy reducido (alrededor de 40), en el contexto de alrededor de 8 mil miembros del sistema. La mitad de estos investigadores de la comunicación es evaluada por el Comité de Ciencias Sociales (que incluye sociología, demografía, economía, ciencia política, etc.) y la otra mitad por el Comité de Humanidades del 
SNI (que incluye antropología, historia, educación, filosofía, etcétera). La elección de área, así como la definición de "disciplina", "subdisciplina" y especialidad es prerrogativa de cada investigador. Si bien es lógico que las "especialidades" difieran, en el catálogo de disciplinas y subdisciplinas del sistema, sólo 14 de los 40 investigadores se identifican con "Comunicación", todos ellos en el área de ciencias sociales, donde está clasificada, asociada con la subdisciplina "Comunicaciones Masivas".

Dado que la mayor parte de estos investigadores desarrolla sus actividades principales de docencia en los programas de posgrado ( $\mathrm{y}$, más específicamente, en los reconocidos por el Padrón de Excelencia), pues la correspondencia institucional es muy alta, sería interesante explorar con mayor detalle, en otro análisis, las implicaciones de esta auto-adscripción disciplinaria para la conformación del campo académico de la comunicación. Un rasgo adicional, significativo en este sentido, es la muy escasa incorporación al SNI de investigadores menores de cuarenta años, pues uno de los requisitos de entrada es el doctorado, y el hecho de que el rápido crecimiento del número de investigadores de la comunicación en el SNI en la última década (en 1993 había menos de la mitad de los actuales) se explique más por la "actualización" de grados académicos de los investigadores ya establecidos, que por la formación de nuevos doctores que, en su caso, aún no ingresan al sistema.

Por otra parte, con estos datos puede reforzarse la idea, que ya se había hecho notar desde años atrás, de que en México los recursos más calificados, los apoyos institucionales y los proyectos académicos más productivos en el campo de la comunicación, se han seguido concentrando en muy pocas universidades, a pesar de que también en ellas enfrentan condiciones poco favorables, especialmente en términos disciplinarios aislados. $\mathrm{Al}$ mismo tiempo, la demanda por estudios de especialización y actualización profesional, atendida con diversos criterios "de mercado" por cada vez más universidades e instituciones no universitarias, ha confundido el carácter educativo de los posgrados, incluyendo el doctorado, de manera coincidente con la tendencia opuesta, de restricción de la calificación de la "excelencia académica".

Persisten como problemas centrales, tanto para los programas acreditados por Conacyt como para los demás, el bajo índice de titulación ("eficiencia terminal"), la escasez de profesores y la insuficiente articulación con líneas institucionales e interinstitucionales de investigación. En términos generales, el diagnóstico de los posgrados mexicanos realizado en 1989, ante un conjunto notablemente mayor de programas, conserva su validez en 2002 . Pero, en un plano más amplio, puede afirmarse que en la última década, como causa y efecto de múltiples factores, la identidad disciplinaria de los estudios de comunicación, especialmente en lo que respecta a la investigación y el posgrado, se ha vuelto mucho más compleja de sostener y es objeto de debate no sólo teórico e intelectual, sino también estratégico en los planos institucional, 
político y profesional. Los posgrados y la investigación de la comunicación, al mismo tiempo que se han consolidado y fortalecido, se han desvinculado de la formación de profesionales y de los enfoques disciplinarios en comunicación que siguen sosteniéndose en las licenciaturas, para avanzar en la integración multidisciplinaria entre las ciencias sociales y las humanidades (Fuentes, 2002).

En este plano, igual a lo que sucede en otros países latinoamericanos y, de alguna manera en Estados Unidos y en Europa, la disyuntiva entre la disciplinarización y la disolución disciplinaria de los estudios de comunicación en México es el desafío fundamental que el campo habrá de seguir enfrentando en la primera década del siglo XXI. Los avances "post-disciplinarios" (Fuentes, 1999) en la investigación podrán desembocar en una u otra de las alternativas, dependiendo de las estrategias adoptadas por sus propios agentes, por los practicantes de otras disciplinas, y sobre todo, por la orientación de las políticas nacionales en el sector universitario y científico, que también están en proceso de cambio, aparentemente radical.

En efecto, a partir de los cambios en el sistema político mexicano derivados de las elecciones de julio de 2000 , se han desencadenado procesos de reestructuración de las políticas públicas en todos los sectores, incluyendo el científico y universitario, cuyos propósitos están declarados pero cuya mecánica de implantación y resultados son todavía inciertos en agosto de 2002 . En el marco del Programa Nacional de Educación 2001-2006 y del Programa Especial de Ciencia y Tecnología 2001-2006, se han modificado los reglamentos, procedimientos y convocatorias del Sistema Nacional de Investigadores y del ahora llamado Programa para el Fortalecimiento del Posgrado Nacional. Este establece, primero, el apoyo a los "Programas Integrales de Fortalecimiento del Posgrado de las Instituciones de Educación Superior (PIFOP), que tengan como objetivo fundamental mejorar la calidad de los programas de posgrado que imparten a nivel de especialidad, maestría y doctorado [...]" y, luego, el "Padrón Nacional de Posgrado, con el propósito de reconocer la buena calidad de los programas de posgrado" (Conacyt-SEP: 2001).

Para estas dos modalidades, se incluyen tanto los "programas de posgrado con orientación profesional" como los "programas de posgrado con orientación a la investigación", cada tipo con un sistema de indicadores de evaluación más preciso que el anteriormente empleado. En el Padrón Nacional de Posgrado se clasificarán los programas como "Competente a nivel internacional" y de "Alto nivel" según, entre otros indicadores, el reconocimiento internacional o nacional de los profesores. (Conacyt-SEP: 2001).

Estos cambios de marcos institucionales, en su escala mayor, sin duda habrán de modificar las condiciones en que se ha desarrollado el campo académico de la comunicación (y todos los demás campos académicos) en México hasta ahora, pero las implicaciones concretas no pueden estimarse 
aún (en agosto de 2002 no se habían publicado los resultados de la primera convocatoria). Mientras tanto, la relevancia de los objetos de estudio identificados con la "comunicación" sigue creciendo, más extensa y rápidamente que la competencia y logros de los "especialistas" en ellos. Y uno de los tipos de productos académicos en que estas características pueden apreciarse mejor es el que constituyen las tesis de posgrado.

\section{Las tesis de maestría como indicadores del futuro del campo}

Se han seleccionado para este análisis las tesis presentadas entre 1996 y 2000 en tres programas de maestría en comunicación que, durante ese periodo, contaban con registro en el Padrón de Excelencia del Conacyt, factor que no sólo implica un reconocimiento a su calidad académica, sino también la dotación de apoyos financieros, especialmente becas para los estudiantes. No se consideraron otros programas inscritos en el padrón (como las maestrías de la UAM-Xochimilco, la Universidad de Guadalajara o el ITESO) sobre todo porque debido a su más reciente fundación, no han producido aún tesis en un número mínimamente considerable; ni otros programas, cuyos objetivos de formación los alejan de los parámetros de la investigación, o que, sea por su orientación o por el tiempo transcurrido, tampoco han generado un conjunto de tesis evaluable como tal.

La Maestría en Comunicación de la Universidad Iberoamericana, adscrita al Departamento de Comunicación, fue la primera establecida en el país y, aunque ha sufrido varias reestructuraciones y reorientaciones desde 1976, había otorgado el grado, en el año 2000, a más de ochenta de sus egresados. En el periodo de análisis, se presentaron ahí 42 tesis. La Maestría en Ciencias de la Comunicación de la Facultad de Ciencias Políticas y Sociales de la UNAM, fundada en 1979, ha pasado igualmente por diversos procesos de rediseño, incluyendo el último de ellos, que a partir del año 2000 la integra al Posgrado en Ciencias Políticas y Sociales, impartido en conjunto por la Facultad y el Instituto de Investigaciones Sociales. De las tesis presentadas entre 1996 y 2000, se analizan 40. Finalmente, la Maestría en Ciencias con Especialidad en Comunicación del ITESM campus Monterrey, abierta en 1994, ha generado en pocos años un número relativamente alto de graduados. En el corpus de análisis para este trabajo se incluyen 44 tesis producidas ahí. En síntesis, se trabaja con 126 tesis de maestría, provenientes en proporciones casi equivalentes, de estos tres programas. La distribución de estas tesis por institución y por año de presentación se expone en el cuadro 2. 
Cuadro 2

Tesis de Maestría en Comunicación, por universidad y año (1996-2000)

\begin{tabular}{|l|c|c|c|c|c|c|}
\hline & 1996 & 1997 & 1998 & 1999 & 2000 & Total \\
\hline UNAM & 7 & 6 & 13 & 6 & 8 & $40(32 \%)$ \\
\hline ULA & 4 & 6 & 13 & 9 & 10 & $42(33 \%$ \\
\hline ITESM & 5 & 8 & 9 & 11 & 11 & $44(35 \%)$ \\
\hline Total & $16(13 \%)$ & $20(16 \%)$ & $35(28 \%)$ & $26(20 \%)$ & $29(23 \%)$ & $126(100 \%)$ \\
\hline
\end{tabular}

Un dato descriptivo formal que puede matizar en algún sentido tanto la "eficiencia terminal" de los programas (criterio central de evaluación por Conacyt) como las características de las tesis, es la gran diferencia detectada en el volumen físico de éstas: mientras que en la UNAM el promedio de páginas de las tesis incluidas en el corpus es de 219 y en la UIA de 190, en el ITESM es de sólo 103. Habrá que recordar al respecto que las tesis han sido tradicionalmente muy valoradas, desde la licenciatura, tanto en la UNAM como en la UIA, mientras que los estudiantes de licenciatura del ITESM (de donde proviene la mayoría de sus estudiantes de maestría), no se titulan mediante la presentación de tesis. Otro de los datos sobre los que puede llamarse la atención es que el número de tesis presentadas por año no crece a partir de 1998, ni en el total de la muestra ni en ninguna de las tres instituciones, y que $60 \%$ de los autores de las tesis son mujeres. Por otro lado, en la muestra se incluyen sólo tres tesis elaboradas "en equipo" (dos personas), todas en la UIA. El resto de las tesis son trabajos individuales.

\section{Cuadro 3}

Tesis de Maestría en Comunicación, por universidad y tema-objeto

\begin{tabular}{|c|c|c|c|c|}
\hline & UNAM & UIA & ITESM & Total \\
\hline Comunicación educativa & 3 & 11 & 11 & $25(20 \%)$ \\
\hline Comunicación organizacional & 0 & 8 & 1 & $9(7 \%)$ \\
\hline Comunicación y cultura & 1 & 1 & 1 & $3(2 \%)$ \\
\hline Comunicación y política & 7 & 4 & 0 & $11(9 \%)$ \\
\hline Comunicación y salud & 1 & 2 & 0 & $3(2 \%)$ \\
\hline Historia de los medios & 6 & 1 & 0 & $7(6 \%)$ \\
\hline Lenguajes mediáticos & 7 & 1 & 1 & $9(7 \%)$ \\
\hline Tecnologias de información & 2 & 5 & 7 & $14(11 \%)$ \\
\hline $\begin{array}{l}\text { Industrias culturales y } \\
\text { representaciones }\end{array}$ & 10 & 3 & 11 & $24(19 \%)$ \\
\hline Oferta/consumo cultural & 2 & 2 & $=4$ & $8(6 \%)$ \\
\hline Estudios de recepción & 1 & 4 & 8 & $13(11 \%)$ \\
\hline
\end{tabular}


En cuanto al contenido, el análisis de este corpus comprendió cinco aspectos: tema-objeto de la tesis, tipo de investigación según su método, fase del proceso de comunicación abordado, medio de comunicación analizado y comunidad o sujetos de referencia. Para cada uno de estos aspectos se definieron categorías analíticas mutuamente excluyentes, por lo que debieron formularse en términos amplios, lo más abarcadores que fuera posible.

Como puede verse, los campos temáticos más atendidos, con alrededor de $20 \%$ de las tesis incluidas en el corpus cada uno, fueron "Comunicación educativa" (que incluye educación para la recepción, educación y medios y enseñanza e investigación de la comunicación), mayoritariamente abordado en la UIA y el ITESM, e "Industrias culturales y representaciones", trabajado sobre todo en la UNAM y el ITESM. Aunque la distribución de temas-objeto por institución es más o menos homogénea, hay sesgos perceptibles en tanto que cada universidad ha definido líneas de investigación en que intenta encuadrar las tesis de posgrado que se producen en ella, dependiendo sobre todo de la orientación en este sentido de sus profesores de planta. Estas dos categorías, más la referida a "Tecnologías de información" (y sus usuarios), dan cuenta de la mitad de los temas-objeto abordados en las tesis de maestría, y han sido trabajadas en las tres universidades.

En cuanto a los tipos de investigación según su método general de abordaje, puede apreciarse un claro predominio de los estudios empíricos en las tres instituciones, al mismo tiempo que una muy baja atención al desarrollo conceptual teórico-metodológico y, notablemente, también a las propuestas de intervención (estratégica y sistemática) en ámbitos de la práctica comunicacional, lo cual suscita una duda sobre la congruencia de los "perfiles de egreso" de las tres universidades, que incluyen claros ingredientes "profesionalizantes", con sus productos objetivos. Los datos del cuadro 4 indican la distribución de las tesis en este aspecto.

\section{Cuadro 4}

Tesis de Maestría en Comunicación, por universidad y tipo de investigación

\begin{tabular}{|l|c|c|c|c|}
\hline & UNAM & UIA & ITESM & Total \\
\hline Empirica & 20 & 27 & 35 & $82(65 \%)$ \\
\hline Documental-bibliográfica & 17 & 9 & 3 & $29(23 \%)$ \\
\hline Teórico-metodológica & 3 & 1 & 0 & $4(3 \%)$ \\
\hline Propuesta de intervención & 0 & 5 & 6 & $11(9 \%)$ \\
\hline
\end{tabular}

Tal predominio de los trabajos con enfoque empírico (65\%) entre las tesis de maestría analizadas puede interpretarse como un avance muy significativo para el campo en cuanto al ejercicio de competencias prácticas de investiga- 
ción concreta, aspecto en el que varios investigadores habían insistido como prioridad desde muchos años atrás. La inscripción de algunas de las tesis, en las tres universidades, en proyectos de investigación más amplios, dirigidos por los profesores-investigadores de planta más experimentados, califica también esta tendencia como muy positiva en el sentido de la formación de nuevos investigadores en las maestrías.

Es claro que cuando las tesis se encuadran en un proyecto mayor, sirven tanto para enriquecer empíricamente esos proyectos como para la formación de nuevos investigadores. No obstante, probablemente en muchos de estos casos se tienda a limitar para los estudiantes el desarrollo de las competencias más generales de la investigación, como el diseño de las estrategias y la elaboración de los marcos teórico-metodológicos. Es decir, aunque la tendencia puede interpretarse en términos de un progresivo alejamiento del predominio del "teoricismo", también puede indicar el riesgo de un acercamiento al "empirismo" acrítico. Si bien no se puede profundizar en esta cuestión sin más datos y contexto, queda establecida la duda, que habría que despejar en otro momento. El cuadro 5 desglosa los métodos empleados en las tesis con enfoque empírico.

\section{Cuadro 5}

Tesis de Maestría en Comunicación, con enfoque empírico, por universidad y método de investigación

\begin{tabular}{|l|c|c|c|c|}
\hline & UNAM & UIA & ITESM & Total \\
\hline Análisis de contenido & 12 & 6 & 11 & $29(35 \%)$ \\
\hline Estrategias multimétodos & 4 & 8 & 7 & $19(23 \%)$ \\
\hline Encuestas y entrevistas & 0 & 4 & 10 & $14(17 \%)$ \\
\hline Análisis de medios o lenguaje & 3 & 1 & 3 & $7(8.5 \%)$ \\
\hline Análisis de estrategias & 1 & 4 & 1 & $6(8 \%)$ \\
\hline $\begin{array}{l}\text { Otros métodos (grupos de discusión, } \\
\text { observación etnográfica, etcétera). }\end{array}$ & 0 & 4 & 3 & $7(8.5 \%)$ \\
\hline
\end{tabular}

Quizá el dato más relevante en este análisis sea el empleo, en cerca de $25 \%$ de las tesis empíricas, de estrategias "multimétodos", es decir, la combinación sistemática de diversos instrumentos de producción de información sobre los objetos de estudio. Este rasgo de "eclecticismo" metodológico ha sido caracterizado como indispensable y puesto en práctica por cada vez mayor número de investigadores de la comunicación en el mundo, y es uno de los "indicadores" más fuertes de las tendencias de fortalecimiento o disolución disciplinaria de los estudios sobre la comunicación. Por otra parte, el predominio de los análisis de contenido como método de investigación en $35 \%$ de los trabajos estudiados, no es una tendencia nueva y tiene que ver, 
entre otros factores, con los costos necesarios para realizarlos, menores por lo general que los correspondientes a estudios "de campo" como las encuestas o la etnografía. A su vez, los análisis de contenido han sido considerados, desde hace al menos cinco décadas, como uno de los métodos "propiamente" comunicacionales en el sentido disciplinario. Llaman también poderosamente la atención las bajas proporciones de los estudios empíricos que se centran en el "análisis de medios o lenguaje", y en el "análisis de estrategias", esto último en contraste con el predominio (ver adelante) de las "estrategias de comunicación" como "fase" estudiada del proceso. Quizá sea que las "estrategias" tiendan a considerarse desde perspectivas de análisis diversas, y que son importantes como objetos de estudio aunque no se cuente con métodos específicos de análisis de su ejercicio social. Esta cuestión merece también, en otro momento, un estudio más detallado y profundo.

\section{Cuadro 6}

Tesis de Maestría en Comunicación, por universidad y fase del proceso abordada

\begin{tabular}{|l|c|c|c|c|}
\hline & UNAM & UIA & ITESM & Total \\
\hline Industria cultural & 2 & 1 & 4 & $7(6 \%)$ \\
\hline Emisor & 4 & 0 & 2 & $6(5 \%)$ \\
\hline Estrategias de comunicación & 3 & 11 & 10 & $24(19 \%)$ \\
\hline Contexto & 0 & 7 & 0 & $7(6 \%)$ \\
\hline Politicas públicas & 3 & 2 & 0 & $5(4 \%)$ \\
\hline Productos y mensajes & 14 & 4 & 6 & $24(19 \%)$ \\
\hline Medios & 7 & 2 & 4 & $13(10 \%)$ \\
\hline Usos de tecnologías & 2 & 5 & 4 & $11(9 \%)$ \\
\hline Recepción y mediaciones & 4 & 5 & 8 & $17(13 \%)$ \\
\hline Consumo cultural & 0 & 1 & 3 & $4(3 \%)$ \\
\hline Representaciones/identidades & 1 & 4 & 3 & $8(6 \%)$ \\
\hline
\end{tabular}

El tercer eje de análisis tiene que ver con la "fase" del proceso de comunicación analizada. Aunque es cada vez menos exhaustivo para dar cuenta de la investigación de la comunicación, el modelo elemental de "producción-circulación-consumo" de mensajes, heredero del más elemental aún de "emisor-mensaje-receptor", sigue sirviendo para distinguir una buena parte de los acercamientos, si bien la proporción de los trabajos que pueden encuadrarse cómodamente en ese esquema es cada vez menor. La "comunicación", conceptualizada desde marcos cada vez más variados y divergentes, no sólo pero también en las maestrías, se va desglosando en "dimensiones" construidas desde lógicas distintas. Parece evidente que la diversidad en el estudio de las partes o "fases" del proceso de la comunicación está directa- 
mente relacionada con la diversidad de temáticas elegidas y con las metodologías empleadas en los proyectos de investigación. En el cuadro 6 pueden verse las categorías empleadas para analizar este aspecto en las tesis estudiadas.

Aquí llama especialmente la atención que el análisis de "productos y mensajes" comunicativos, una manifestación de las tradiciones disciplinarias más centrales en la investigación de la comunicación, mediante los análisis de contenido sobre todo, predomine en la misma medida que el análisis de "estrategias de comunicación", que a su vez implica una contextualización social e institucional de las prácticas comunicativas, más relacionada con tendencias "post-disciplinarias". La primera categoría se concentra sobre todo en la UNAM y la segunda en el ITESM y la UIA. Los estudios sobre "recepción y mediaciones" (que, para estos efectos pueden sumarse a los de "consumo cultural" y a los de "usos de tecnologías"), siguen superando ampliamente en número a los dedicados a la exploración de la "producción" "emisión" o circulación social de mensajes.

En estricto paralelo con la categorización anterior, la clasificación de las tesis según el "medio" de comunicación estudiado presenta también un carácter ambiguo: aunque siguen siendo reconocibles en un buen porcentaje de los casos los estudios sobre "medios" (al grado que la mitad de las tesis tienen que ver con televisión, Internet o prensa), también son altos los porcentajes de trabajos en que no se estudia ningún "medio" o, no siendo éste el objeto principal, se trata sobre varios de ellos o sobre "combinaciones nuevas". La televisión es el medio "favorito" en el ITESM, Internet en la UIA y la prensa en la UNAM. El cuadro 7 expone los resultados de este análisis.

\section{Cuadro 7}

Tesis de Maestría en Comunicación, por universidad y medio de comunicación estudiado

\begin{tabular}{|l|c|c|c|c|}
\hline & UNAM & UIA & ITESM & Total \\
\hline Televisión & 9 & 7 & 14 & $30(24 \%)$ \\
\hline Video & 2 & 2 & 2 & $6(5 \%)$ \\
\hline Cine & 4 & 0 & 2 & $6(5 \%)$ \\
\hline Radio & 1 & 2 & 1 & $4(3 \%)$ \\
\hline Prensa & 10 & 3 & 6 & $19(15 \%)$ \\
\hline Medios impresos (revistas, folletos) & 2 & 1 & 1 & $4(3 \%)$ \\
\hline Internet, computadora, satélite & 3 & 17 & 5 & $25(20 \%)$ \\
\hline Varios medios (organizacionales & 1 & 8 & 1 & $10(8 \%)$ \\
\hline Otros medios & 5 & 0 & 1 & $6(5 \%)$ \\
\hline Ningún medio & 6 & 9 & 1 & $16(12 \%)$ \\
\hline
\end{tabular}


Finalmente, se categorizaron las comunidades o sujetos de referencia en las tesis, en tanto agentes institucionales o personales de la comunicación sometida a estudio. La distribución de las tesis según este aspecto refuerza la ambivalencia de las tendencias encontradas en los análisis precedentes. Las "industrias culturales" son las entidades sociales más estudiadas, pero en la UNAM se concentra claramente el estudio de los "emisores" y en el ITESM el de los "receptores y audiencias" o "usuarios". El Estado parece haber dejado de ser una entidad interesante para los estudiantes de maestría (o para los investigadores que dirigen las tesis), en comparación con décadas anteriores. Esta distribución se puede ver en el cuadro 8.

\section{Cuadro 8}

Tesis de Maestría en Comunicación, por universidad y comunidades o sujetos de referencia

\begin{tabular}{|l|c|c|c|c|}
\hline & UNAM & UIA & ITESM & Total \\
\hline Industrias culturales & 15 & 4 & 12 & $31(25 \%)$ \\
\hline Emisores & 12 & 4 & 2 & $18(14 \%)$ \\
\hline Emisores y receptores & 1 & 5 & 2 & $8(6 \%)$ \\
\hline Receptores y audiencias & 4 & 7 & 17 & $28(22 \%)$ \\
\hline Usuarios & 0 & 6 & 8 & $14(11 \%)$ \\
\hline Estado & 3 & 3 & 0 & $6(5 \%)$ \\
\hline Organización/institución educativa & 0 & 10 & 1 & $11(9 \%)$ \\
\hline Otros & 5 & 3 & 2 & $10(8 \%)$ \\
\hline
\end{tabular}

Si bien se han subrayado aquí sólo algunos aspectos del análisis, pueden identificarse diferencias sustanciales en los tres programas de posgrado comparados mediante las tesis producidas en el lapso estudiado. Se puede hablar, en términos generales, más que de dispersión, de un grado alto de diversidad (o de diversificación) temática y teórico-metodológica en las investigaciones analizadas, relacionado en parte con las opciones institucionales por ciertas perspectivas y las líneas de investigación desarrolladas por su personal académico de planta, pero también con tendencias más generales de constitución del campo académico de la comunicación. En este sentido se detectan también fuertes similitudes entre las tres maestrías, clara y protagónicamente centradas en el campo nacional.

Muchas de las tesis analizadas, al final, mencionan que la investigación en cuestión es tan sólo una "aproximación" al tema y proponen una lista de problemas que podrían ser abordados en otras investigaciones. Pero, con pocas excepciones, las tesis parecen responder más a preocupaciones individuales de sus autores que a esas propuestas de continuidad. Las excepciones están asociadas a los proyectos más amplios en que se encuadran varias tesis, 
como es el caso de las agendas de la prensa latinoamericana o la oferta y consumo de televisión, en el ITESM, o la historia de la prensa nacional, en la UNAM. Como en otros productos de investigación académica de la comunicación en México, en las tesis se detecta una escasa (y en muchos casos nula) atención a los antecedentes nacionales de investigación. Si acaso, los "estados de la cuestión" toman como puntos de partida sustanciales los aportes realizados en otros países, no en México. La razón más obvia es la escasísima circulación y lectura, en el propio ámbito académico especializado, de los productos -cuyo número y calidad crecen constantemente- de los esfuerzos nacionales en el campo.

Entre las tesis presentadas en la UIA, cerca de la mitad son estudios de caso o propuestas de productos o procesos de comunicación en un lugar y un tiempo específicos, que probablemente tengan aplicación práctica. Muchas de las tesis del ITESM consisten, a su vez, en estudios puntuales de la oferta y/o el consumo de productos comunicacionales (sobre todo audiovisuales), o en investigaciones descriptivas de uso de tecnologías o desarrollo de productos mediados. En contraste, las tesis de la UNAM suelen ser más "teóricas" y tienden a analizar situaciones socioculturales y económico-políticas macro. En las tres instituciones se nota un esfuerzo por asociar las investigaciones de tesis con subproductos publicables en forma de artículos en revistas académicas (tanto impresas como electrónicas), pero éstas, a su vez, tienen una circulación extremadamente restringida. No puede hablarse, todavía, de que las investigaciones realizadas bajo la modalidad de tesis de maestría aporten al conocimiento y a la constitución del campo académico, independientemente del nivel de rigor, precisión o pertinencia de sus marcos teórico-metodológi$\cos$, en donde hay grandes diferencias. Muchas de las tesis analizadas terminan siendo, todavía, un ensayo pobremente fundamentado o un producto comunicativo inconsistente con su propio marco teórico-metodológico. De ahí la duda, también, respecto a la formación de nuevos investigadores en proporción a las necesidades estimadas en el campo o a los recursos invertidos. También hay, por supuesto, tesis ejemplares, que merecerían una mayor difusión y análisis que los que reciben. Habría que alentar a sus autores, y en muchos casos así se hace, a continuar el trayecto y profundizar el cultivo de sus competencias académicas en el doctorado.

La estructura institucional que soporta al campo académico de la comunicación en México se encuentra, todavía o nuevamente, en una fase de transición delicada. Las tendencias en tensión (y algunas, en franca contradicción) no permiten proyectar certezas sobre el futuro, aunque sí preguntas críticas, que habrá que responder en la discusión y en la práctica cotidianas. La exploración analítica de las tesis de maestría producidas recientemente en tres de los programas más prestigiados y sólidos en el campo nacional, puede ayudar a identificar algunos indicios o manifestaciones tanto de los factores 
que generan sus características presentes como de los rasgos que habrá que buscar que se conviertan en predominantes en las próximas décadas. Independientemente de las "brechas" generacionales, institucionales, geográficas o de género que lo atraviesan, el campo académico de la comunicación requiere un conocimiento auto-analítico mucho mayor que el disponible. Las reuniones nacionales de responsables de posgrados, convocadas por el CONEICC entre 1999 y 2001, no lograron generar el "diagnóstico" percibido colectivamente como indispensable, ni formular las colaboraciones consensuales que parecían posibles para el futuro común, al menos en términos comparativos con el marco elaborado en 1989. Es de esperarse una recuperación institucional de mayor alcance en los años por venir.

\section{Bibliografía}

Bourdieu, Pierre (1972), Esquisse d'une théorie de la pratique, Ginebra, Droz. - (1975), "La spécificité du champ scientifique et les conditions sociales du progrès de la raison", en Sociologie et Sociétés, París, vol. vII, núm. 1, pp. 91-118.

(1988), Homo Academicus, California, Stanford University Press. (2000), Los usos sociales de la ciencia, Buenos Aires, Nueva Visión.

Conacyt-SEP (2001), Convocatoria 2001-2002 del Programa para el Fortalecimiento del Posgrado Nacional. Publicado el 31 de octubre de 2001 en Internet.

[http://www.main.conacyt.mx/daic/padron-excel/index.html]

Condit, Celeste Michelle (1989), "Replacing Oxymora: Instituting Communication Studies", en Dervin et al. (eds.), Rethinking Communication, vol. 1, Newbury Park, CA, Sage, pp. 154-156.

Fuentes Navarro, Raúl (1988), La investigación de comunicación en México. Sistematización documental 1956-1986, México, Ediciones de Comunicación.

(1996), La investigación de la comunicación en México. Sistematización documental 1986-1994, Guadalajara, ITESO-UdeG.

- (1998), La emergencia de un campo académico. Continuidad utópica y estructuración científica de la investigación de la comunicación en México, Guadalajara, ITESO-UdeG.

- (1999): "La investigación de la comunicación en América Latina: condiciones y perspectivas para el siglo XXI", en Diá-logos de la Comunicación, Lima, FELAFACS, núm. 56, pp. 52-68. 
- (2000), "Educación y telemática", en Enciclopedia Latinoamericana de Sociocultura y Comunicación, Buenos Aires, Grupo Editorial Norma, núm. 6 .

(2002): "El campo académico de la comunicación en México: fundamentos de la postdisciplinariedad", en Valenzuela (coord.), Los estudios culturales en México, México, FCE (en prensa).

- y Enrique E. Sánchez Ruiz (1989), “Algunas condiciones para la investigación científica de la comunicación en México", en Huella, Guadalajara, núm. 17.

- (1992), "Investigación sobre comunicación en México: los retos de la institucionalización", en Orozco (coord.), La investigación de la comunicación en México: tendencias y perspectivas para los noventas, México, Cuadernos de Comunicación y Prácticas Sociales, Universidad Iberoamericana, núm. 3, p.11-38.

Jara Elías, José Rubén (1981), “Información básica sobre la investigación de la comunicación en México: documentos, instituciones, publicaciones, investigadores y un análisis del estado actual de la disciplina", en Comunicación, algunos temas, México, Cenapro-Armo, año 1, núms. 2, 3 y 4.

Juárez Mendías, Rosa Esther (1989), Relatoría final de la Primera Reunión Nacional de Posgrados y Centros de Investigación en Comunicación, Guadalajara, ITESO.

Luna Cortés, Carlos E. (1989), "El posgrado en comunicación: una fuga hacia arriba", en Renglones, Guadalajara, ITESO, núm. 14, pp. 61-64.

Rota, Josep (1974), "Remarks on Journalism Education and Research in the Americas", en Mass Communication in Mexico, México, Universidad Iberoamericana-Association for Education in Journalism.

Sánchez Ruiz, Enrique E. (comp.) (1988), La investigación de la comunicación en México. Logros, retos y perspectivas, México, Ediciones de Comunicación-UdeG. 